\title{
Review
}

Mario Plebani*

\section{Clinical laboratory: bigger is not always better}

https://doi.org/10.1515/dx-2018-0019

Received May 3, 2018; accepted May 11, 2018; previously published online May 29, 2018

\begin{abstract}
Laboratory services around the world are undergoing substantial consolidation and changes through mechanisms ranging from mergers, acquisitions and outsourcing, primarily based on expectations to improve efficiency, increasing volumes and reducing the cost per test. However, the relationship between volume and costs is not linear and numerous variables influence the end cost per test. In particular, the relationship between volumes and costs does not span the entire platter of clinical laboratories: high costs are associated with low volumes up to a threshold of 1 million test per year. Over this threshold, there is no linear association between volumes and costs, as laboratory organization rather than test volume more significantly affects the final costs. Currently, data on laboratory errors and associated diagnostic errors and risk for patient harm emphasize the need for a paradigmatic shift: from a focus on volumes and efficiency to a patientcentered vision restoring the nature of laboratory services as an integral part of the diagnostic and therapy process. Process and outcome quality indicators are effective tools to measure and improve laboratory services, by stimulating a competition based on intra- and extra-analytical performance specifications, intermediate outcomes and customer satisfaction. Rather than competing with economic value, clinical laboratories should adopt a strategy based on a set of harmonized quality indicators and performance specifications, active laboratory stewardship, and improved patient safety.
\end{abstract}

Keywords: costs; diagnostic errors; outcome measures; prices; quality indicators; volume.

\footnotetext{
*Corresponding author: Prof. Mario Plebani, Dipartimento Strutturale Medicina di Laboratorio, Azienda Ospedale Università di Padova Via Giustiniani, 2 - 35128 Padova, Italy; and Department of Medicine-DIMED, Medical School, University of Padova, Padova, Italy, Phone: 0498212792, E-mail: mario.plebani@unipd.it. http://orcid.org/0000-0002-0270-1711
}

\section{Introduction}

Laboratory services around the world are undergoing substantial consolidation and changes through mechanisms ranging from mergers, acquisitions and outsourcing, primarily based on expectations to improve efficiency, increasing volumes and reducing the cost per test [1]. However, there has been little evaluation of the risks that these changes have on quality and patient safety. The aim of this paper is to discuss available data on laboratory consolidation and outsourcing.

\section{Volume-outcome relationship in health care}

The primary argument used to promote laboratory consolidation is that merging may improve outcomes through increased volume. However, important caveats in the relationship between volume-outcome should be taken into consideration. In medicine, the volume-outcome relationship varies widely across conditions and outcomes, with the largest benefits occurring among a small number of technically difficult surgical interventions, such as esophagectomy and pancreatectomy. For most other conditions, the benefits of volume are less pronounced and the volume-outcome relationship is usually not linear [2]. Emerging evidence highlights that volume may be simply a proxy for other processes, such as having systems in place to recognize and effectively manage complications. Revising the evidence of the literature, Tsai and Jha suggested that improvement in delivering high-quality care should be achieved focusing on improving processes that create better outcomes for patients and stated that "relying on increased volume to create quality may be confusing cause and effect" [3]. More than a decade ago, a remarkable study found that the volume of lung resections performed in a hospital was a better predictor of mortality after pancreatoduodenectomies than the volume of pancreatoduodenectomies, suggesting that the story of the volume-outcome ratio is a bit more complicated than "practice makes perfect" [4]. Unfortunately the seminal vision by Michael Porter that "health care is shifting focus 
from the volume of services delivered to the value created for patients, with 'value' defined as the outcomes achieved relative to the costs" [5] is still poorly translated in practice. Progress is slow and halting, partly because outcomes measurements remain limited, not standardized and focused on clinical but not functional status [6]. However, moving from volumes to value measured with reliable sets of outcomes represents a decisive step in accelerating value improvement in health care. Currently, the transformation from volume to value is no longer theoretical and requires meaningful quantification of costs and outcomes at the level of individual patients [7].

\section{Volume-outcome relationship in laboratory medicine}

In laboratory medicine, it has been demonstrated that the relationship between volume and costs is not linear and that numerous variables influence the end cost per test. In particular, the relationship between volumes and costs does not span the entire platter of clinical laboratories: high costs are associated with low volumes up to a threshold of 1 million test per year. Over this threshold, there is no linear association between volumes and costs, as laboratory organization rather than test volume more significantly affects the final costs. Laboratory organization, the complexity of the main organization (e.g. accademic centers, primary, secondary or tertiary health care centers), different case-mix and the inpatients to outpatients ratio, as well as other management issues play a relevant role in assuring efficiency, and should be taken into account when planning projects for reorganizing the delivery of laboratory services in the healthcare system [8]. Another study, performed in a different geographic area (Taiwan), confirmed that increasing test volume is not enough to achieve efficiency as enhancing the level of quality and test specialization are further requirements to survive in an increasing competitive environment [9]. In addition, the relationship between costs and prices paid to clinical laboratories is a controversial issue as they do not appear to be evidence-based. As a matter of fact, the prices for laboratory tests vary widely within and across countries. In the US, a large distribution of prices for the 10 most commonly used diagnostic tests was reported, as shown in Table 1.

Table 1 presents the distribution of prices paid per test by a US supermarket chain (Safeway) to different clinical laboratories in 2010. Across all 10 tests, the 95th percentile price exceeded the 5 th percentile price by an average factor of 10; the price for the most commonly prescribed
Table 1: Distribution of prices paid by Safeway across laboratories in 2010 for the 10 most commonly used diagnostic tests (from Ref. [10], modified).

\begin{tabular}{lrrr}
\hline Laboratory test & \multicolumn{3}{c}{ Percentile, $\$$} \\
\cline { 2 - 4 } & 5th & 50th & 95th \\
\hline Panel & & & \\
Basic metabolic & 5.75 & 17.15 & 126.44 \\
General health & 20.58 & 23.88 & 121.86 \\
Comprehensive metabolic & 7.18 & 15.98 & 132.48 \\
Lipid & 8.85 & 11.73 & 74.92 \\
Hepatic function & 5.56 & 11.32 & 85.14 \\
Iron test & 4.40 & 4.71 & 58.47 \\
Total prostate-specific antigen & 12.50 & 13.36 & 88.75 \\
Thyroxin free test & 6.13 & 8.19 & 64.00 \\
Thyroid-stimulated hormone & 11.42 & 28.53 & 101.70 \\
Uric acid test & 3.07 & 3.47 & 30.60 \\
\hline
\end{tabular}

test, the basic metabolic panel, ranged from $\$ 5.75$ to \$126.44, the highest price 22 times higher than the lowest. Similar data have been reported by a different insurer in North Carolina and before the implementation of reference pricing [10]. In many countries, the adoption of the diagnosis-related group (DRG) system, designed to reimburse the overall fee for the entire period of hospitalization based on the patient's diagnosis, overcomed the traditional feefor-service reimbursement, that was based on reimbursing the costs of each and all laboratory tests performed. The fee-for-service system of provider payment is increasingly viewed as an obstacle to achieving effective, coordinated and efficient care [11]. However, for inpatients the feefor-service system is still used for reimbursing clinical laboratories even if bundled payment (BP) arrangements, designed to remunerate multiple providers for coordinating the total amount of services required for a single, pre-defined episode of care are claimed [12]. Bundled payment may contain comprehensive diagnostics and therapy, including laboratory, pathology and surgery, as well as routine follow-up at regular intervals [13]. A global fee for "care episodes" has been advocated to reduce the request of unnecessary tests and services, to reward care coordination and efficiency as well as to reduce variation in payment for longitudinal care of patients with chronic conditions [14]. BP should strongly affect the models of laboratory services, shifting the focus from volumes and cost per test to the effectiveness of laboratory information in improving diagnostic and therapeutic pathways, as well as clinical and economical outcomes. This, in turn, should promote efforts to improve appropriateness in test request, while moving away from "distorted economics" that have forced laboratories to follow the production industry 
model. Indeed, this may confirm that laboratory testing is to be considered part of the diagnostic and therapeutic processes, thus precluding the risk of identifying laboratory data as a commodity to be purchased. Therefore, in laboratory medicine, available evidence highlights that the relationship between volume and costs is not linear, numerous variables influence the end cost per test. In addition, data collected in our institution show that direct costs (instrumentation, reagents, consumable and personnel) represent about half per cent of the total costs, as indirect overhead costs such as administrative staff, hospital operations, information technology and maintenance represent almost $40 \%$ of total costs. Even when total costs are included in the calculation, the profitability of a mediumsize laboratory remains above $200 \%$ [15]. If volumes are not representative of costs, more intriguing and controversial is the relationship between volume and quality.

\section{Volume-quality relationship in laboratory medicine}

Quality in laboratory medicine is a never-ending quest, being defined as "an unfinished journey". After focusing on internal indicators of analytical quality and efficiency, thanks to the evidence of the vulnerability of extra-analytical phases and the need for a more patient-centered approach, efforts are shifting toward indicators of total quality, clinical effectiveness and patient outcomes with an emphasis on the benefit created for patients by "value", defined as the "outcomes achieved relative to costs" [16]. As we stated elsewhere, "quality in laboratory medicine should be defined as the guarantee that each and every step in the total testing process is correctly performed, thus ensuring valuable decision making and effective patient care" [17]. In addition, clinical laboratories must also provide evidence of value in test-treatment pathways, as well as in improving clinical outcomes and patient safety [15]. Quality in laboratory medicine, therefore, has two dimensions, which can no longer be separated. The "internal dimension", performed and assured within the laboratory environment to provide efficiency, is based on accuracy and reliability of analytical results, timeliness in their production and communication and, finally, on cost-containment activities. The "external dimension" is assured by diagnostic accuracy, value in test-treatment pathways, effect on clinical and economical outcomes and, finally, on patient safety. In fact, the effectiveness of a test depends on not only the result being delivered according to set standards (e.g. accuracy, timeliness and acceptable costs) but also the result being acted upon appropriately and in a timely fashion to assure valuable outcomes [18].

Although no evidence-based and robust data are available to demonstrate the relationship between different volumes and quality of laboratory services, some lessons should come from the outsourcing experiences. Outsourcing of services as potential cost-saving measures began with nonmedical services such as food and laundry, but has spread to medical services such as pharmacy, radiology and the clinical laboratory [19]. The main goal of outsourcing laboratory testing is to decrease the cost per test thanks to the huge volumes performed by large health care companies. A number of studies have examined the issue of clinical laboratory outsourcing providing data on related benefits and problems [20, 21]. In particular, experiences in US academic centers have been evaluated through an anonymous online survey. The results obtained show that: (a) over time, the financial gains fell short of expectations as cost savings eroded as initially favorable test pricing was followed in later years by price increases; (b) there was a lack of utilization control incentive with facilitated excessive testing, excessive send outs and excessive costs; (c) the remaining on-side rapid response laboratory experienced an increasing workload but reduced significance in the eyes of the hospital administration; (d) lack of clinician satisfaction both for poor turnaround time as well as for lack of available expert consultation supporting test requests and interpretation of results; (e) inconsistent and nonreproducible results, particularly in molecular pathology, flow cytometry and microbiology leading to poor patient care [22]. In addition, a significant negative impact on educational programs, difficulties in organizing departmental conferences and teaching activities have been reported. Medical errors arising from outsourcing laboratory and radiology services have been reported as resulting from inappropriate turnaround times for test reports, compromised specimens, incorrect test and problems with sample transportation [23, 24]. Other articles reported the possible negative outcomes in the case of the removal of laboratory services to a distant location as this should cause major disruptions in communication and prevent the accomplishment of the educational mission, particularly in hospitals that support residency and fellowship programs [25].

\section{Toward an outcome-based evaluation of clinical laboratory services}

The state-of-the-art in laboratory medicine highlights on the one hand, impressive improvements in automation, 
throughput, and workflow, and on the other hand, the vulnerability of extra-analytical phases, the association between errors in test request and result interpretation with diagnostic errors and the increasing evidence of the potential risk for patient safety due to excessive and inappropriate testing $[26,27]$. As a matter of fact, the focus on internal indicators of analytical quality, efficiency and cost-per test led to overlooking the value of laboratory services in improving patient outcomes and population health. In fact, although it was stated that "the value of a laboratory test must be ascertained not only on the basis of its chemical or clinical performance characteristics, but also by its impact on patient management, the only true assessment of the quality of testing being quality of patient outcomes" [28], the link between laboratory quality and patient outcomes has proven to be elusive as clinical outcomes reflect the combined activities of clinicians, nurses, laboratory and radiology professionals, other healthcare professionals as well as the patient [29]. In the last years, however, several research lines emphasized some fundamental issues to provide better laboratory services. First, laboratory process quality should begin and end "outside the laboratory", extending quality measurement to steps in the total testing process antecedent to the analytical step (pre-pre-analytical phase) and subsequent to result reporting (post-post-analytical phase) [30, 31]. Second, appropriate stewardship of laboratory resources may improve patient care by ensuring the correct tests are performed at the appropriate time while unnecessary tests are avoided. At the end of the testing process, appropriate test interpretation and utilization should be pursued, as shown in Figure 1 [32, 33]. The figure represents the concept of clinical laboratory stewardship in the context of the wellknown "brain-to-brain cycle" emphasizing the critical issues of the laboratory-clinical interface [34]. A fundamental step of the clinical pathology stewardship which results from interventions to improve test ordering and result interpretation, is the identification of reliable quality indicators for monitoring all steps of the testing process, particularly the extra-analytical phases. Monitoring of process-based quality indicators has been found an effective tool to improve laboratory performances documenting a significant decrease in defect rates [35]. The identification of a set of harmonized quality indicators covering all steps of the testing process and the definition of related performance specifications $[36,37]$ represent fundamental steps toward a more patient-centered evaluation of the ultimate quality of laboratory services. However, some global measures of laboratory testing value have been recently proposed: (a) broadening test process turnaround time to time-to-diagnosis, as initiating high quality care is typically dependent on achieving a diagnosis; (b) broadening result report accuracy to diagnostic accuracy, as an accurate diagnosis is a prerequisite for an optimum treatment decision and is often predicated on, or supported by, laboratory information; (c) adding a new intermediate outcome that is diagnostic completeness as laboratory information may reveal not only the cause of patient's condition but also relevant contextual factors (e.g. genetic factors) that influence the treatment choice [29].

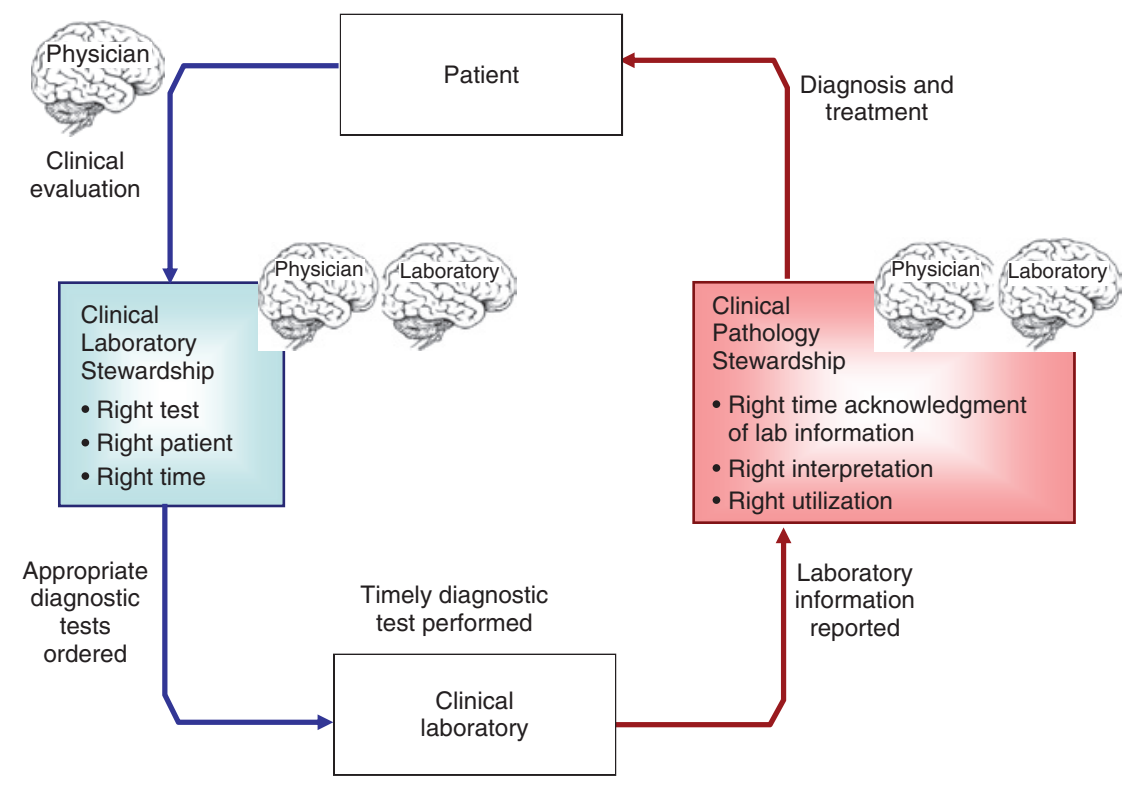

Figure 1: Clinical laboratory stewardship (from Ref. [18], modified). 


\section{Conclusions}

In the last few decades, laboratory medicine has undergone monumental changes and the landscape of clinical laboratories is still evolving over time. In particular, data on laboratory errors and associated diagnostic errors and risk for patient harm $[38,39]$ emphasize the need for a paradigmatic shift: from a focus on volumes and efficiency to a patient-centered vision restoring the nature of laboratory services as an integral part of the diagnostic and therapy process. Process and outcome quality indicators are effective tools to measure and improve laboratory services by stimulating a competition based on intra- and extra-analytical performance specifications, intermediate outcomes and customer satisfaction. Some internal and external drivers for this paradigmatic change have been described already [18] and are based on the re-evaluation of the clinical-laboratory interface to reduce the risk of over- and under-testing, the search for a more appropriate interpretation and utilization of the laboratory information in the clinical context and better clinical and economical outcomes. Some years ago, a strategy to overcome current inefficiencies in the delivery of laboratory services was designed. It should encompass translation into integrated, multidisciplinary care, in which appropriateness in test request and interpretation both play a central role in assuring efficiency and effectiveness [40]. After 7 years, evidence has been accumulated to confirm that vision, and more data and tools are available to move that wishful thinking a reality. But it still time to conclude that "blessed are those who have not seen and yet have believed" (John 19:29).

Author contributions: The author has accepted responsibility for the entire content of this submitted manuscript and approved submission.

Research funding: None declared.

Employment or leadership: None declared.

Honorarium: None declared.

Competing interests: The funding organization(s) played no role in the study design; in the collection, analysis, and interpretation of data; in the writing of the report; or in the decision to submit the report for publication.

\section{References}

1. Plebani M. The changing face of clinical laboratories. Clin Chem Lab Med 1999;37:711-7.

2. Livingston EH, Cao J. Procedure volume as a predictor of surgical outcomes. J Am Med Assoc 2010;304:95-7.
3. Tsai TC, Jha AK. Hospital consolidation, competition, and quality: is bigger necessarily better? J Am Med Assoc 2014;312:29-30.

4. Jha AK. Back to the future: volume as a quality metric. J Am Med Assoc 2015;314:214-5.

5. Porter ME. What is value in health care? N Engl J Med 2010;363:2477-81.

6. Porter ME, Larsson S, Lee TH. Standardizing patient outcomes measurement. N Engl J Med 2016;374:504-6.

7. Lee VS, Kawamoto K, Hess R, Park C, Young J, Hunter C, et al. Implementation of a value-driven outcomes program to identify high variability in clinical costs and outcomes and association with reduced cost and improved quality. J Am Med Assoc 2016;316:1061-72.

8. Barletta G, Zaninotto M, Faggian D, Plebani M. Shop for quality or quantity? Volumes and costs in clinical laboratories. Clin Chem Lab Med 2013;51:295-301.

9. Su BG, Chen SF, Yeh SH, Shih PW, Lin CC. Cost evaluation of clinical laboratory in Taiwan's National Health System by using activity-based costing. Clin Chem Lab Med 2016;54:1753-8.

10. Robinson JC, Whaley C, Brown TT. Association of reference pricing for diagnostic laboratory testing with changes in patient choices, prices, and total spending for diagnostic tests. JAMA Intern Med 2016;176:1353-9.

11. Institute of Medicine. Rewarding provider performance: aligning incentives in Medicare. Washington, DC: National Academies Press, 2006.

12. Plebani M. Clinical laboratories: production industry or medical services? Clin Chem Lab Med 2015;53:995-1004.

13. Herzlinger RE, Schleicher SM, Mullangi S. Health care delivery innovations that integrate care? Yes!: but integrating what? J Am Med Assoc 2016;315:1109-10.

14. Davis K. Paying for care episodes and care coordination. N Engl J Med 2007;356:1166-8.

15. Lippi G, Plebani M. The add value of laboratory diagnostics: the many reasons why decision-makers should actually care. J Lab Precis Med 2017;12:1-4.

16. Plebani M. Analytical quality: an unfinished journey. Clin Chem Lab Med 2018;56:357-9.

17. Plebani M. Quality indicators to detect pre-analytical errors in laboratory testing. Clin Biochem Rev 2012;33:85-8.

18. Plebani M. Quality and future of clinical laboratories: the Vico's whole cyclical theory of the recurring cycles. Clin Chem Lab Med 2018;56:901-8.

19. Lee J. Evaluating lab outsourcing. Hospitals seek savings but have to consider quality, service and staff issues. Mod Healthc 2014;44:22-4.

20. Feiberg B, Kaden PA. Pros and cons of outsourcing laboratory services. J Oncol Pract 2006;2:162-3.

21. Forsman RW. Joint venture versus outreach: a financial analysis of case studies. Clin Leadersh Manag Rev 2001;15:217-21.

22. Mrak RE, Parslow TG, Tomaszewski JE. Outsourcing of academic clinical laboratories: experiences and lessons from the association of pathology chairs laboratory outsourcing survey. Acad Pathol 2018;5:1-5.

23. Chasin BS, Elliott SP, Klotz SA. Medical errors arising from outsourcing laboratory and radiology services. Am J Med 2007;120:819.e9-11.

24. Poon EG, Gandhi TK, Sequist TD, Murff HJ, Karson AS, Bates DW. "I wish I had seen this test result earlier!": dissatisfaction with 
test result management systems in primary care. Arch Intern Med 2004;164:2223-8.

25. Procop GW, Winn W. Outsourcing microbiology and offsite laboratories. Implications on patient care, cost savings, and graduate medical education. Arch Pathol Lab Med 2003;127:623-4.

26. Plebani M. Laboratory-associated and diagnostic errors: a neglected link. Diagnosis 2014;1:89-94.

27. Plebani $M$. The detection and prevention of errors in laboratory medicine. Ann Clin Biochem 2010;47:101-10.

28. Plebani M. Charting the course of medical laboratories in a changing environment. Clin Chim Acta 2002;319:87-100.

29. Epner PL. Appraising laboratory quality and value: what's missing? Clin Biochem 2017;50:622-4.

30. Plebani M. Towards a new paradigm in laboratory medicine: the five rights. Clin Chem Lab Med 2016;54:1881-91.

31. Meier AF, Badrick TC, Sikaris KA. What's to be done about laboratory quality? process indicators, laboratory stewardship, the outcomes problem, risk assessment, and economic value: responding to contemporary global challenges. Am J Clin Pathol 2018;149:186-96.

32. Messacar K, Parker SK, Todd JK, Dominguez SR. Implementation of rapid molecular infectious disease diagnostics: the role of diagnostic and antimicrobial stewardship. J Clin Microbiol 2017;55:715-23.

33. Dickerson JA, Fletcher AH, Procop G, Keren D, Singh IR, Garcia JJ, et al. Transforming laboratory utilization review into laboratory stewardship: guidelines by the PLUGS National Committee for Laboratory Stewardship. J Appl Lab Med 2017;2:259-68.
34. Plebani M, Laposata M, Lundberg GD. The brain-to-brain loop concept for laboratory testing 40 years after its introduction. Am J Clin Pathol 2011;136:829-33.

35. Meier FA, Souers RJ, Howanitz PJ, Tworek JA, Perrotta PL, Nakhleh RE, et al. Seven Q-Tracks monitors of laboratory quality drive general performance improvement: experience from the College of American Pathologists Q-Tracks program 1999-2011. Arch Pathol Lab Med 2015;139: 762-75.

36. Sciacovelli L, Panteghini M, Lippi G, Sumarac Z, Cadamuro J, Galoro $C A$, et al. Defining a roadmap for harmonizing quality indicators in Laboratory Medicine: a consensus statement on behalf of the IFCC Working Group "Laboratory Error and Patient Safety" and EFLM Task and Finish Group "Performance specifications for the extra-analytical phases". Clin Chem Lab Med 2017;55:1478-88.

37. Plebani M. EFLM Task Force on Performance Specifications for the extra-analytical phases. Performance specifications for the extra-analytical phases of laboratory testing: Why and how. Clin Biochem 2017;50:550-4.

38. Plebani M. Errors in clinical laboratories or errors in laboratory medicine? Clin Chem Lab Med 2006;44:750-9.

39. Plebani M, Lippi G. Improving diagnosis and reducing diagnostic errors: the next frontier of laboratory medicine. Clin Chem Lab Med 2016;54:1117-8.

40. Plebani M, Lippi G. Is laboratory medicine a dying profession? Blessed are those who have not seen and yet have believed. Clin Biochem 2010;43:939-41. 